ARTICLE

\title{
SCF Fbxo22-KDM4A targets methylated p53 for degradation and regulates senescence
}

Yoshikazu Johmura, ${ }^{1 \star}$, Jia Sun ${ }^{1, \star}$, Kyoko Kitagawa ${ }^{2}$, Keiko Nakanishi ${ }^{3}$, Toshiya Kuno ${ }^{4}$, Aya Naiki-Ito ${ }^{4}$, Yumi Sawada', Tomomi Miyamoto ${ }^{5}$, Atsushi Okabe ${ }^{6}$, Hiroyuki Aburatani ${ }^{6}$, ShengFan Li ${ }^{7}$, Ichiro Miyoshi ${ }^{5}$, Satoru Takahashi ${ }^{4}$, Masatoshi Kitagawa ${ }^{2} \&$ Makoto Nakanishi ${ }^{1}$

Recent evidence has revealed that senescence induction requires fine-tuned activation of $\mathrm{p} 53$, however, mechanisms underlying the regulation of p53 activity during senescence have not as yet been clearly established. We demonstrate here that SCFFbxo22-KDM4A is a senescenceassociated E3 ligase targeting methylated p53 for degradation. We find that Fbxo22 is highly expressed in senescent cells in a p53-dependent manner, and that $\mathrm{SCF}^{\mathrm{Fbx} 022}$ ubiquitylated p53 and formed a complex with a lysine demethylase, KDM4A. Ectopic expression of a catalytic mutant of KDM4A stabilizes p53 and enhances p53 interaction with PHF20 in the presence of $\mathrm{Fbxo22}$. SCF $\mathrm{Fbx}^{22}-\mathrm{KDM} 4 \mathrm{~A}$ is required for the induction of p16 and senescenceassociated secretory phenotypes during the late phase of senescence. Fbxo22 $2^{-/-}$mice are almost half the size of $\mathrm{FbxO}_{2} 2^{+/-}$mice owing to the accumulation of $\mathrm{p} 53$. These results indicate that $\mathrm{SCF}^{\mathrm{Fb} \times 022}-\mathrm{KDM} 4 \mathrm{~A}$ is an $\mathrm{E} 3$ ubiquitin ligase that targets methylated p53 and regulates key senescent processes.

\footnotetext{
${ }^{1}$ Department of Cell Biology, Graduate School of Medical Sciences, Nagoya City University, 1 Kawasumi, Mizuho-cho, Mizuho-ku, $467-8601$ Nagoya, Japan.

${ }^{2}$ Department of Molecular Biology, Hamamatsu University School of Medicine, Higashi-ku, 431-3192 Hamamatsu, Japan. ${ }^{3}$ Department of Perinatology, Aichi Human Service Center, Institute for Developmental Research, 713-8 Kamiya-cho, Kasugai, Aichi 489-0392, Japan. ${ }^{4}$ Department of Experimental Pathology and Tumor Biology, Graduate School of Medical Sciences, Nagoya City University, 1 Kawasumi, Mizuho-cho, Mizuho-ku, 467-8601 Nagoya, Japan.

${ }^{5}$ Department of Comparative and Experimental Medicine and Center for Animal Sciences, Graduate School of Medical Sciences, Nagoya City University, 1 Kawasumi, Mizuho-cho, Mizuho-ku, 467-8601 Nagoya, Japan. ${ }^{6}$ Genome Science Division, Research Center for Advanced Science and Technology, The University of Tokyo, Meguro-ku, 153-8904 Tokyo, Japan. ${ }^{7}$ Zhongshan Hospital of Dalian University, 6 Jiefang St, Zhongshan District, 116001 Dalian, China. * These authors contributed equally to this work. Correspondence and requests for materials should be addressed to M.N.

(email: mkt-naka@med.nagoya-cu.ac.jp).
} 
A $\mathrm{n}$ important hallmark of senescence is the inability to proliferate in response to physiological mitotic stimuli ${ }^{1}$. The limited lifespan of human cells is governed by telomere length ${ }^{2,3}$ as well as various genotoxic stressors, all of which ultimately activate DNA-damage responses ${ }^{4}$. We and others have recently uncovered a molecular mechanism involved in permanent cell cycle arrest during the senescence process in which p53 activation at G2 has a necessary and sufficient role by inducing a mitosis skip ${ }^{5,6}$. Another hallmark of senescence is the appearance of senescence-associated secretory phenotypes (SASP), such as robust secretion of numerous growth factors, cytokines, proteases and other proteins, which can cause deleterious effects on the tissue microenvironment ${ }^{7}$. On the other hand, SASP also has positive effects on the repair of damaged tissue, at least at a young age ${ }^{8}$. Induction of these two hallmarks of senescence is often coordinated, but their respective mechanisms do not always overlap. Most notably, p38MAPK is critically required for SASP through activating $N F-\kappa B$ independent of canonical DDR, but p53 restrains p38MAPK leading to the suppression of SASP in senescent cells ${ }^{9}$. There appear to be missing links that could more fully explain the antagonistic effects of p53 on the induction of these two representative hallmarks of senescence.

The key to the regulation of p53 activity is control of the stability of its protein, which is mainly orchestrated through a network of ubiquitylation reactions ${ }^{10,11}$, although other mechanisms such as regulation of its localization are also involved $^{12,13}$. While numerous E3 ubiquitin ligases for p53 have been reported ${ }^{14}$, data are less clear regarding the in vivo relevance of these E3 ligases in p53 regulation except for murine double minute 2 (Mdm2; refs 15,16). Mdm2 is itself a transcriptional target of $\mathrm{p} 53$, and acts to create a negative feedback loop ${ }^{17}$. Importantly, in mice with a disrupted p53$\mathrm{Mdm} 2$ feedback loop, the degradation profile of p53 upon DNA damage appeared to be normal ${ }^{18}$, suggesting the role of $\mathrm{Mdm} 2$ as the sole E3 ubiquitin ligase for stress-induced p53 into question.

Several lines of evidence have clearly indicated that posttranscriptional modification of p53 also has a critical role in the regulation of its activity ${ }^{11,19}$. For example, DNA-damage-induced phosphorylation of p53 at Ser15 stabilizes and activates p53, suppressing Mdm2-mediated p53 ubiquitylation ${ }^{20}$. Acetylation or methylation of lysine residues located at the C-terminal domain (CTD) of p53 is also reported to regulate p53 activity ${ }^{21,22}$. Although acetylation at the CTD is indispensable for p53 activation, methylation appears to vary in the degree to which it is required according to both the location and extent of the methylation state ${ }^{23}$. More importantly, the effect of the interplay between acetylation and methylation at the CTD of p53 is largely unknown.

Fbxo22 is not yet a well-characterized F-box protein. It was first identified as a p53-targeting gene ${ }^{24}$, then was later reported to form a complex with KDM4 whose degradation regulates histone H3 methylation at lysines 9 and 36 (ref. 25). Here, we identify the $\mathrm{SCF}^{\mathrm{Fbxo22}}$-KDM4A complex as an E3 ubiquitin ligase for methylated $\mathrm{p} 53$ and show that upon senescence-inducing stimulation, $\mathrm{SCF}^{\mathrm{Fbxo22}}-\mathrm{KDM} 4 \mathrm{~A}$ is required for induction of $\mathrm{p} 16$ and SASP in senescent cells.

\section{Results}

Fbxo22 is highly expressed in senescent cells. We have recently uncovered the molecular basis of senescence induction, which results at least in part from generation of tetraploid G1 cells by mitosis skipping 5 . In order to determine the factor(s) that regulate senescent processes, we first tried to identify the genes that are predominantly expressed in larger sized senescent cells with tetraploid DNA (Fig. 1a and Supplementary Fig. 1a,b). The P1 fraction predominantly exhibited typical senescent phenotypes (SA- $\beta$-gal-positive and flattened morphology), whereas the P2 fraction did not (Fig. 1b,c). Global expression analysis using sorted larger sized cells treated with IR (10 Gy) revealed that 33 genes were expressed at levels fourfold greater than in normalsized cells (Supplementary Fig. 1c). IR treatment of normal human fibroblast HCA2 cells revealed that Fbxo22 as well as WIPI-1, PPP2R5C, and DARC were markedly induced at relatively later time points when compared with $\mathrm{Hdm} 2$ (a human Mdm2 homologue) and p21 (Fig. 1d). Thus, the results suggested that our screening preferentially isolated more slowly induced genes after senescence-inducing stimulation. Induction of Fbxo22 in response to IR was dependent on the presence of p53 since p53 depletion in RPE cells almost completely abolished its induction at both RNA and protein levels (Fig. 1e). Again, induction of Fbxo22 appeared markedly slower when compared with $\mathrm{Hdm} 2$ and $\mathrm{p} 21$. Thus, these results indicated that Fbxo22 is a transcriptional target of p53 as reported previously ${ }^{24}$. Consistent with this, Fbxo22 possesses two p53 binding consensuses on its promoter region (Supplementary Fig. 2, left panels). ChIP-seq analysis of the region around the Fbxo22 gene confirmed recruitment of p53 to the transcription start site of this gene in the presence of 5FU in HCT116 $p 53^{+/+}$ cells, but not in the isogenic $p 53^{-/-}$cells (Supplementary Fig. 2, right panels). This recruitment of p53 was associated with an increase in the H3K27Ac level and the extension of phosphoRNA polymerase II (P-RNAPII).

Fbxo22 regulates the abundance of $\mathrm{p} 53$ and cell proliferation. When RPE cells were depleted of Fbxo22 using doxycyclineinducible Fbxo22 shRNA, the levels of p53 and p21 were significantly increased (Fig. 2a). These inductions were effectively suppressed by re-introduction of wild-type Fbxo22, eliminating the possibility that the influence of Fbxo22 depletion resulted from its off-target effect. Although KDM4A was reported to be targeted for proteosomal degradation by Fbxo22 (ref. 25), the level of KDM4A was not affected in RPE cells by Fbxo22 depletion independent of the p53 status (Fig. 2a). Fbxo22 depletion resulted in a severe defect in cell proliferation, which was effectively recovered by re-introduction of wild-type Fbxo22 or simultaneous depletion of p53 (Fig. 2b). The impaired cell proliferation appeared to be owing to accumulation of cells at G1 phase (Supplementary Fig. 3a, four left panels). In contrast, overexpression of Fbxo22 resulted in the loss of p53 and p21 (Fig. 2c). These effects were not observed when its F-box $(\Delta \mathrm{F})$, FIST-C $(\Delta$ FIST-C) or FIST-N $(\Delta$ FIST-N) domain-deleted mutants were expressed. Hence, overexpression of wild-type Fbxo22, but not $\Delta \mathrm{F}, \Delta$ FIST-C or $\Delta$ FIST-N mutants, enhanced cell proliferation (Fig. 2d) owing to the reduction in the population of G1 cells (Supplementary Fig. 3a, four right panels). Although treatment with Nutlin 3a markedly induced p53, p21 and Hdm2 in RPE cells, ectopic Fbxo22 strongly suppressed their respective inductions even under inhibition of $\mathrm{Hdm} 2$ or $\mathrm{Hdm} 2$ depletion (Supplementary Fig. 3b,c), indicating that the suppression of p53 by Fbxo22 is independent of $\mathrm{Mdm} 2$. Immunohistochemical analyses revealed that this protein predominantly localized in the nucleus (Supplementary Fig. 3d).

SCF ${ }^{\text {Fbxo22 }}$ ubiquitylates p53. Cycloheximide treatment revealed that the half-life of p53 appeared to be around $10 \mathrm{~min}$ in control RPE cells, whereas it was around $60 \mathrm{~min}$ in Fbxo22-depleted RPE cells (Fig. 3a). Thus, Fbxo22 likely regulates p53 stability under unperturbed conditions. Indeed, treatment with the proteasome inhibitor, MG132, almost completely abolished 
Fbxo22-dependent reduction of p53 protein (Supplementary Fig. 4a). Using non-denatured extracts, a greatly enhanced ubiquitylation signal was detected on p53 in the presence of Fbxo22 (Fig. 3b). Similarly, under denatured IP conditions, a strong ubiquitylation signal was detected in the presence of wildtype Fbxo22, but not its mutants lacking the F-box or FIST-C domain (Fig. 3c and Supplementary Fig. 4b). Consistent with this, ectopic expression of wild-type Fbxo22, but not its mutant lacking FIST-C, facilitated the ubiquitylation of p53 in RPE cells (Fig. 3d). In order to investigate whether Fbxo22 could mediate K48-linked polyubiquitylation (a major form of degradationassociated ubiquitylation ${ }^{26}$ ), we transfected HEK293 cells with plasmids expressing a mutant ubiquitin in which lysine 48 was replaced by arginine (K48R) or in which all lysines were replaced except for lysine 48 in a ubiquitin molecule (K48) as well as plasmids expressing 6xMyc-p53 and Flag-Fbxo22. Cells were then treated with MG132 and the ubiquitylation of p53 was analysed.
The Fbxo22-mediated ubiquitylation signal was readily detected in cells expressing K48 ubiquitin but not in those expressing K48R (Supplementary Fig. 4c). Thus, our results suggest that $\mathrm{SCF}^{\mathrm{Fbxo22}}$ mainly promotes K48-linked ubiquitylation of $\mathrm{p} 53$. Taken together, these results indicate that SCF ${ }^{\text {bxoo22 }}$ acts as an E3 ubiquitin ligase in targeting p53 for degradation.

Fbxo22 binds to the unacetylated CTD of p53. Ectopically expressed Flag-Fbxo22 in RPE cells formed a stable complex with p53 in the presence of MG132, whereas this complex was not detected in the absence of MG132 (Fig. 4a). Endogenous Fbxo22 also formed a complex with p53 in RPE cells when treated with MG132 (Fig. 4b). Although the F-box protein often interacts with its substrates through a phosphorylation-dependent degron ${ }^{27}$ and multiple sites of p53 are phosphorylated in response to DNA damage ${ }^{20}$, Fbxo22 equally bound to 553 treated with or without IR and phosphatase (Fig. 4c). Interestingly, complex formation

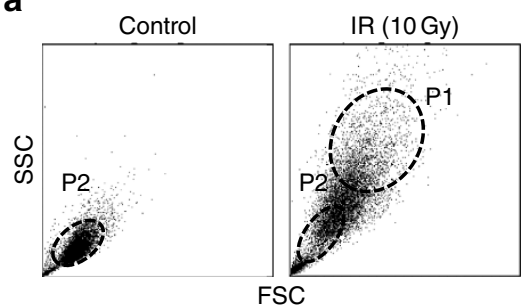

b

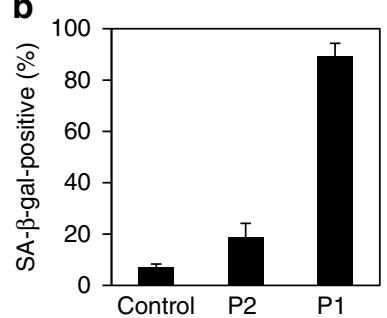

C

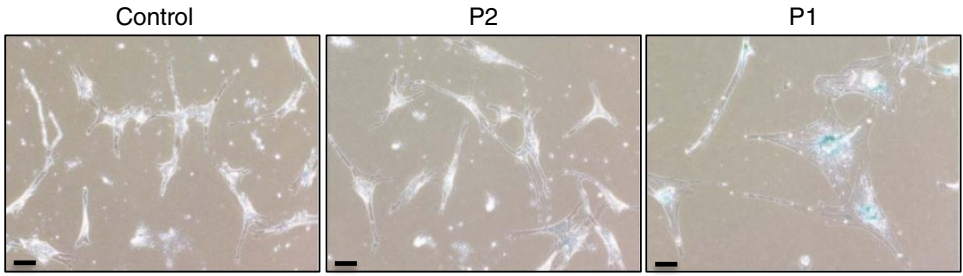

d IR (10Gy)

e Time after $\frac{\text { shControl }}{2} \longrightarrow$ shp53
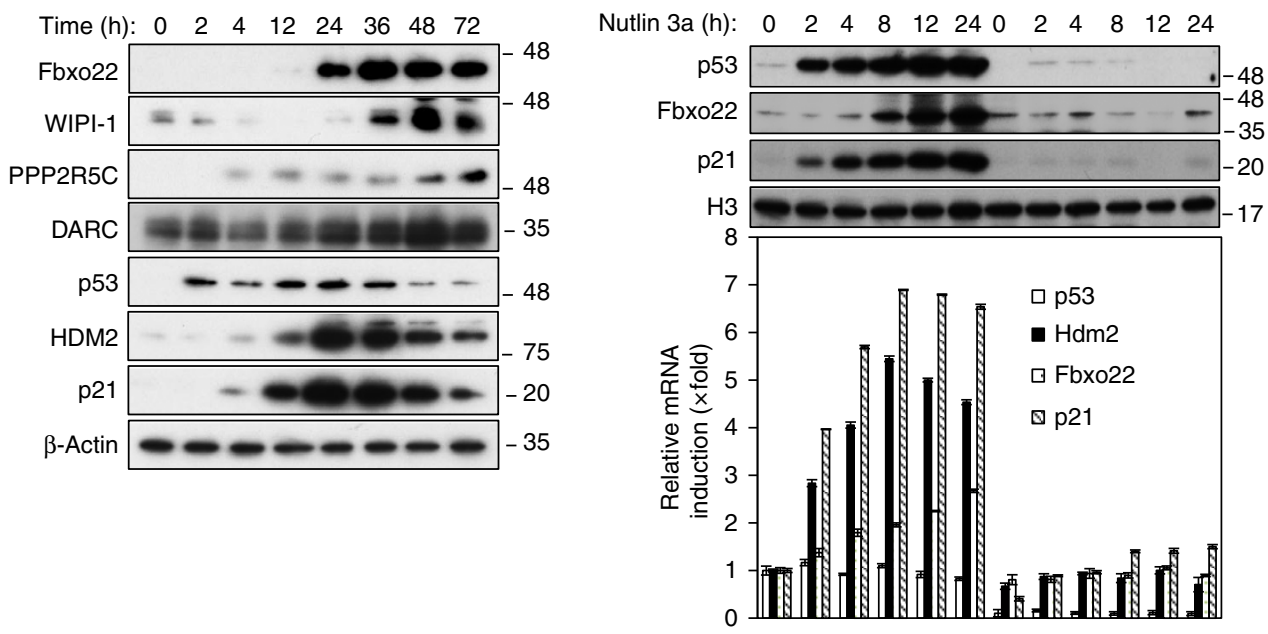

Figure 1 | Identification of Fbxo22 as a protein predominantly expressed in larger sized senescent cells and regulation of its expression by p53. (a) HCA2 cells at $72 \mathrm{~h}$ after IR treatment (10 Gy) were sorted according to size and structure (FSC and SSC) and fractionated as P1 and P2 by FACScan. In the absence of IR (Control), the vast majority of cells were fractionated into the P2 fraction. (b) HCA2 cells at $72 \mathrm{~h}$ after IR irradiation (10 Gy) were sorted by FSC and SSC into P1 and P2 fractions as shown in Fig. 1a. The sorted cells, as well as HCA2 cells without IR irradiation as a control, were cultured for an additional 3 days, and then subjected to an SA- $\beta$-gal assay. (c) Representative images of cells in $\mathbf{b}$ are shown. (d) Lysates from HCA2 cells collected at the indicated times after IR (10 Gy) treatment were subjected to immunoblotting using the indicated antibodies. (e) RPE cells expressing Dox-inducible control shRNA (shControl) or p53 shRNA (shp53) were treated with doxycycline ( $1 \mu \mathrm{g} \mathrm{ml}^{-1}$ ). Cell lysates (upper panels) or total RNAs (lower panels) of

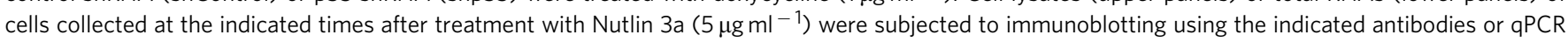
analysis, respectively. Data are presented as means \pm s.d. of at least three independent experiments. 
a

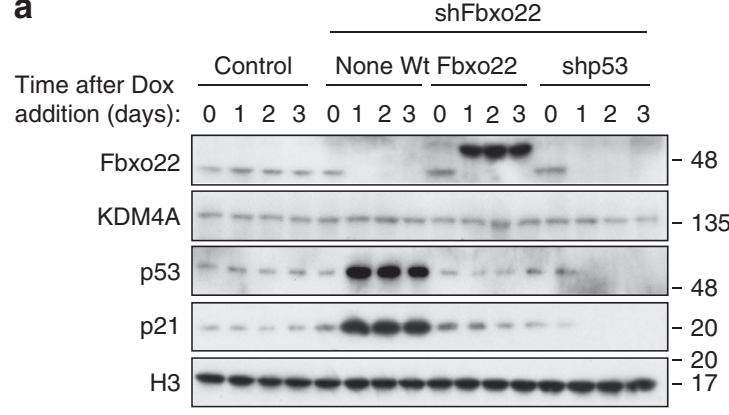

C

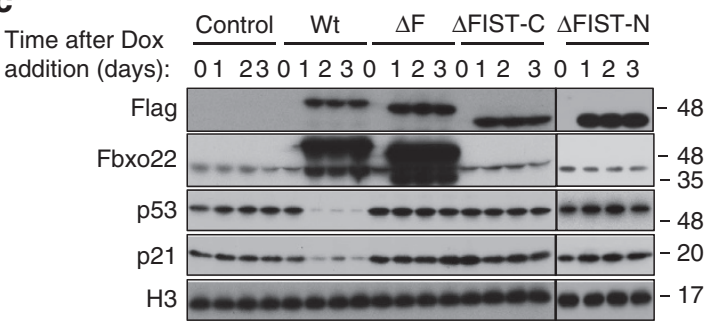

b

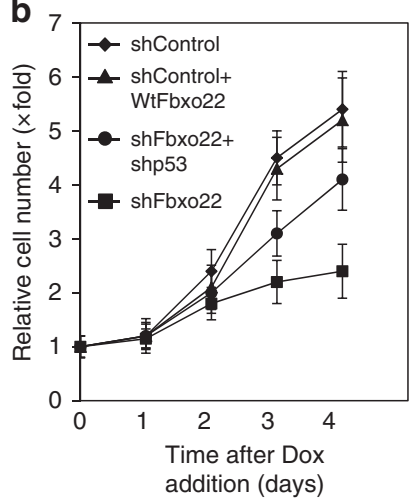

d

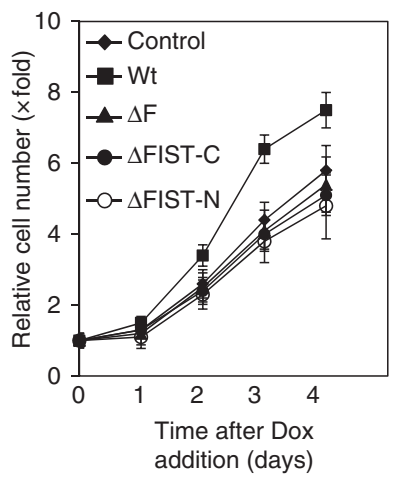

Figure 2 | Fbxo22 controls cell proliferation through regulation of the p53 level. (a) RPE cells expressing the Dox-inducible shControl or shFbxo22 with or without either Dox-inducible Fbxo22 or shp53 were treated with doxycycline $\left(1 \mu \mathrm{g} \mathrm{ml}^{-1}\right)$. Lysates of cells collected at the indicated times were subjected to immunoblotting using the indicated antibodies. (b) The relative numbers of cells indicated as in a were determined at the indicated times. (c) RPE cells expressing Dox-inducible wild-type Fbxo22 (Wt), its mutants lacking the F-box domain $(\Delta \mathrm{F})$, FIST-C $(\Delta$ FIST-C) or FIST-N ( $\Delta$ FIST-N), as well as control RPE cells (Control), were treated with doxycycline $\left(1 \mu \mathrm{g} \mathrm{ml}{ }^{-1}\right)$. Lysates of cells collected at the indicated times were subjected to immunoblotting using the indicated antibodies. Because of the antibody epitope, Fbxo22 antibodies failed to recognize $\Delta$ FIST-C and $\Delta$ FIST-N proteins. (d) The relative numbers of cells as in $\mathbf{c}$ were determined at the indicated times. Data are presented as means \pm s.d. of at least three independent experiments.

between Fbxo22 and p53 was significantly suppressed when RPE cells were treated with an HDAC inhibitor, trichostatin A, suggesting that acetylation of p53 inhibited their interaction (Fig. 4d). Since most acetylation sites of p53 are located at the CTD, we speculated whether Fbxo22 could bind to the CTD of p53. As expected, Fbxo22 effectively bound to wild-type p53, but not to its CTD-deleted mutant $(\Delta 30$; Fig. $5 \mathrm{a})$. In order to further confirm that acetylation of p53 CTD prevents complex formation between Fbxo22 and p53, we generated mutants harbouring an acetyl-mimic substitution ( $\mathrm{K}$ to $\mathrm{Q}$ ) at each or all lysines on the $\mathrm{CTD}$ and tested their abilities to bind to Fbxo22. Although the $\mathrm{K}$ to $\mathrm{Q}$ substitution at each lysine residue did not affect $\mathrm{p} 53$ binding to Fbxo22, the substitution at all lysines (5KQ) almost completely abolished their interactions (Fig. 5b). Interestingly, the $\mathrm{K}$ to $\mathrm{A}$ substitution, but not the $\mathrm{K}$ to Q substitution, at all five lysines as well as at two lysine sites at which p53 was methylated did not affect their interactions, suggesting that substitution at these lysines did not result in marked conformational changes in the p53 CTD domain. These results also suggest that Fbxo22 likely bound to the methylated p53 but failed to bind to the acetylated p53 (Supplementary Fig. 5). We then examined the effect of methylation at these lysines on Fbxo22-dependent degradation of p53. Intriguingly, methylation of p53 at K370 was greatly increased in Fbxo22-depleted RPE cells in the presence of MG132, whereas acetylation at this site was not (Fig. 5c). Taken together, the results strongly suggest that $\mathrm{SCF}^{\mathrm{Fbxo} 22}$ preferentially targeted methylated p53, but not acetylated p53, in ubiquitindependent degradation.
SCF ${ }^{\text {Fbxo22 }}$ forms a ternary complex with p53 and KDM4A. Fbxo22 contains F-box, FIST-N and FIST-C domains (Fig. 6a). p53 bound to the wild type and to a mutant lacking FIST-C, but not to a mutant lacking FIST-N (Fig. 6b), indicating that FIST-N of Fbxo22 is a p53-binding site for this protein. Given that previous mass spectrometry revealed that Fbox 22 formed a stable complex with $\mathrm{KDM} 4 \mathrm{~A}^{25}$ and that $\mathrm{SCF}^{\mathrm{Fbxo} 22}$ preferentially targeted methylated $\mathrm{p} 53$, we speculated that $\mathrm{SCF}^{\mathrm{Fb} x 022}$ might form a ternary complex with p53 and KDM4A. Wild-type Fbxo22 and its mutant lacking FIST-N bound to KDM4A, whereas the mutant lacking FIST-C failed to do so (Fig. 6b), indicating that the FIST-C domain of Fbxo22 is a KDM4A-binding site. Fbxo22 interacted with the SCF complex through its F-box domain, showing that the N-terminal portion of Fbxo22 (1-100) was sufficient for the interaction with Skp1 (Supplementary Fig. 6a). In order to confirm that Fbxo22, p53 and KDM4A form a ternary complex, we performed sequential immunoprecipitation using an anti-Flag M2 affinity gel, followed by elution using Flag peptide and then carried out a second immunoprecipitation using antiKDM4A antibodies. p53 was readily detectable in Flag/KDM4A immunoprecipitates (Fig. 6c). Importantly, the interaction between p53 and KDM4A required Fbxo22 (Supplementary Fig. 6b), indicating that Fbxo22 functions as a scaffold for p53 and KDM4A.

We then asked whether KDM4A demethylase activity is required for $\mathrm{SCF}^{\mathrm{Fbxo22}}$-dependent p53 destabilization. Ectopic expression of wild-type KDM4A did not cause p53 to accumulate in RPE cells, whereas that of a mutant KDM4A lacking 


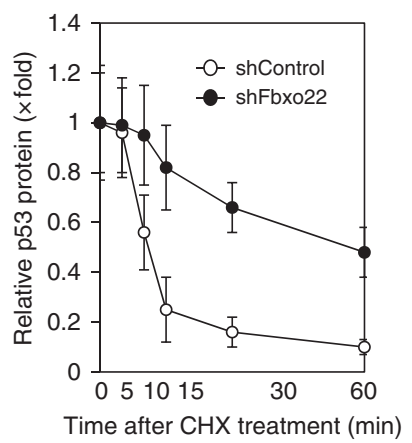

C

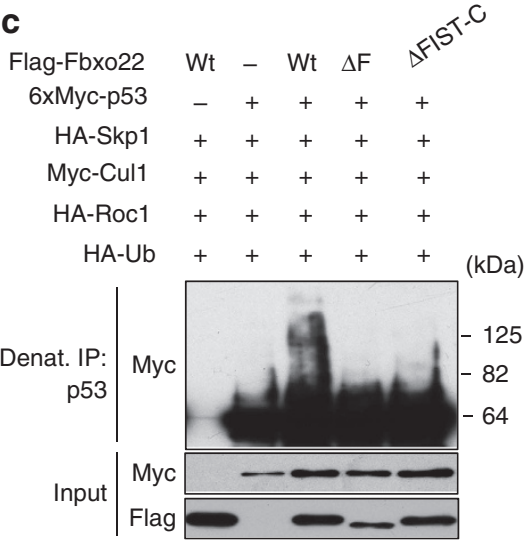

b

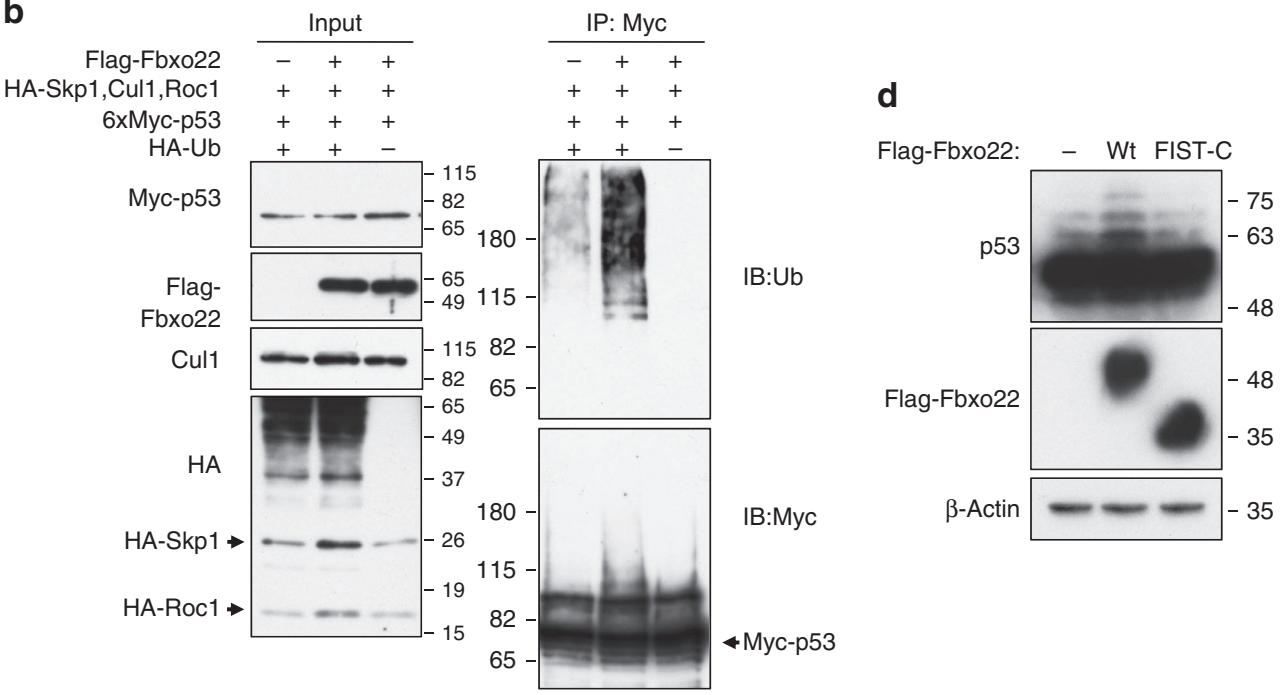

Figure 3 | SCF ${ }^{\text {Fbxo22 }}$ ubiquitylates p53. (a) RPE cells expressing the Dox-inducible shControl or shFbxo22 in the presence of doxycycline $\left(1 \mu \mathrm{g} \mathrm{ml}^{-1}\right)$ for $48 \mathrm{~h}$ were treated with $50 \mu \mathrm{g} \mathrm{ml}^{-1}$ cycloheximide ( $\mathrm{CHX}$ ). Lysates of cells collected at the indicated times were subjected to immunoblotting using the indicated antibodies and the relative p53 intensities were determined using Image J. Data are presented as means \pm s.d. of at least three independent experiments. (b) U2OS cells were transfected with the indicated expression plasmids including SCF components, and were then incubated with MG132 $\left(20 \mu \mathrm{g} \mathrm{ml}^{-1}\right)$ for $5 \mathrm{~h}$. Total cell lysates were subjected to immunoprecipitation with the indicated antibodies under native conditions. The resultant precipitates and lysates (Input) were subjected to immunoblotting using the indicated HRP-conjugated antibodies. (c) To prevent the detection of ubiquitylation of both E3 ligase itself and p53-associated proteins, the cell lysates were immunoprecipitated with anti-p53 antibody under denaturing conditions. The resultant immunoprecipitates and lysates (Input) were subjected to immunoblotting using the indicated HRP-conjugated antibodies. (d) RPE cells expressing the Dox-inducible full-length Flag-Fbxo22 (Wt) or its mutant lacking the FIST-C domain (FIST-C), as well as control RPE cells were incubated in the presence of doxycycline $\left(1 \mu \mathrm{g} \mathrm{ml}{ }^{-1}\right)$ for $48 \mathrm{~h}$ and then treated with MG132 $\left(10 \mu \mathrm{g} \mathrm{ml}^{-1}\right)$ for $6 \mathrm{~h}$. Cell lysates were subjected to immunoblotting using the indicated antibodies.

demethylase activity (H188A) markedly increased the p53 level (Fig. 6d). This increase was not affected by Fbxo22 depletion, although its depletion per se increased the amount of p53 to a level similar to that in cells expressing the mutant KDM4A. In addition, expression of the mutant KDM4A (H188A), but not the wild type, resulted in a specific increase in methylated, but not acetylated, p53 in the presence of MG132, suggesting that KDM4A likely demethylates p53 (Supplementary Fig. 6c). Taken together, $\mathrm{SCF}^{\mathrm{Fbx} 022}$-mediated $\mathrm{p} 53$ degradation requires the demethylase activity of KDM4A. Consistent with this, depletion of KDM4A in RPE cells resulted in a severe defect in cell proliferation (Fig. 6e), owing to the increase in the expression levels of p53 and p21, because co-depletion of p53 effectively reversed impaired cell proliferation in KDM4A-depleted cells (Supplementary Fig. 6d).

Recently, PHF20 was reported to bind to doubly dimethylated p53, stabilizing and activating p53 (ref. 28). When Fbxo22 was depleted from RPE cells, the interaction between ectopically expressed Flag-PHF20 and p53 was greatly enhanced (Fig. 6f). This interaction was completely abolished when two amino acids within the Tudor domain of PHF20 were replaced with alanine. Furthermore, when the demethylase-deficient mutant of KDM4A was expressed, the interaction between PHF20 and p53 was greatly enhanced (Supplementary Fig. 7a). The enhanced interaction was not further affected by Fbxo22 depletion (Supplementary Fig. 7b), indicating that KDM4A functions together with Fbxo22. Depletion of PHF20 suppressed induction of p53 and p21 on IR treatment (Supplementary Fig. 7c). Taken together, these results strongly suggest that $\mathrm{SCF}^{\mathrm{Fbxo22}}-\mathrm{KDM} 4 \mathrm{~A}$ targets methylated p53 for ubiquitin-dependent protein degradation. Acetylation of p53 and PHF20 interaction with dimethylated p53 compete with this ubiquitylation (Fig. 6g).

Fbxo22 is required for induction of p16 and SASP. We then determined the physiological relevance of the negative feedback between $\mathrm{p} 53$ and $\mathrm{SCF}^{\mathrm{Fb} \times 22}-\mathrm{KDM} 4 \mathrm{~A}$ in senescence. For this 
a

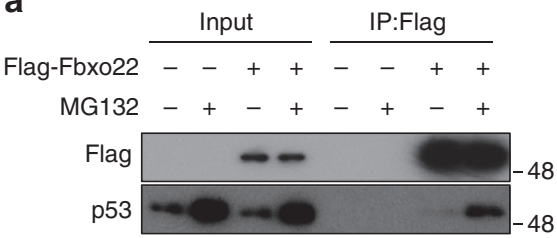

C

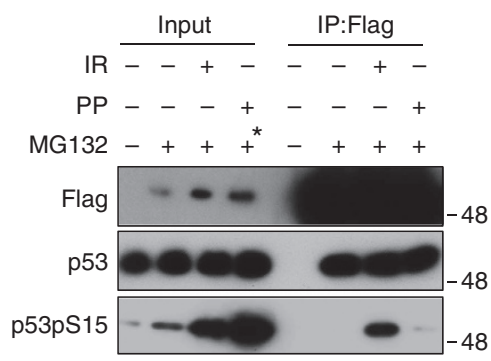

b

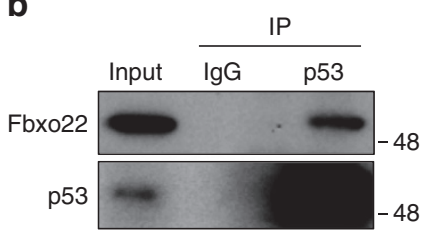

d

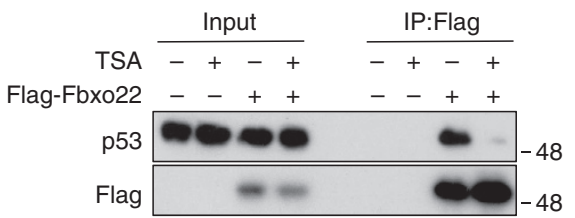

Figure 4 I p53 interacts with Fbxo22 in a phosphorylation-independent manner. (a) RPE cells expressing Dox-inducible Flag-Fbxo22 were incubated for $48 \mathrm{~h}$ in the presence or absence of doxycycline $\left(1 \mu \mathrm{g} \mathrm{ml}{ }^{-1}\right)$ and were then treated with MG132 $\left(10 \mu \mathrm{gl}^{-1}\right)$ for $2 \mathrm{~h}$. The cell lysates were immunoprecipitated using anti-Flag M2 affinity gel. The resultant immunoprecipitates and lysates (Input) were subjected to immunoblotting using anti-Flag or anti-p53 antibodies. (b) RPE cells were treated with MG132 $\left(10 \mu \mathrm{g} \mathrm{ml}^{-1}\right)$ for $2 \mathrm{~h}$ and the lysates were subjected to immunoprecipitation using the anti-p53 antibody or a control lgG. The resultant immunoprecipitates and lysates (Input) were subjected to immunoblotting using the indicated antibodies. (c) Phosphorylation of p53 did not affect its interaction with Fbxo22. RPE cells expressing Dox-inducible Flag-Fbxo22 were incubated for $48 \mathrm{~h}$ in the presence or absence of doxycycline $\left(1 \mu \mathrm{g} \mathrm{ml}^{-1}\right)$ and were then treated with or without MG132 $\left(10 \mu \mathrm{g} \mathrm{ml} \mathrm{I}^{-1}\right)$ for $2 \mathrm{~h}$ and/or with IR (6 Gy). The lysates were immunoprecipitated using anti-Flag M2 affinity gel. After immunoprecipitation, some samples were treated with protein phosphatase (PP) for $1 \mathrm{~h}$. The resultant immunoprecipitates and lysates (Input) were subjected to immunoblotting using the indicated antibodies. The asterisk indicates an input sample before PP treatment. (d) Interaction between Fbxo22 and p53 was suppressed by treatment with TSA. RPE cells expressing Dox-inducible Flag-Fbxo22 were incubated for $48 \mathrm{~h}$ in the presence or absence of doxycycline $\left(1 \mu \mathrm{g} \mathrm{ml} \mathrm{l}^{-1}\right)$ and were then treated with or without TSA (1 mM) for $12 \mathrm{~h}$ and/or MG132 $\left(10 \mu \mathrm{gl}^{-1}\right)$ for $2 \mathrm{~h}$. The cell lysates were immunoprecipitated using anti-Flag M2 affinity gel. The resultant immunoprecipitates as well as lysates (Input) were subjected to immunoblotting using the indicated antibodies.

a

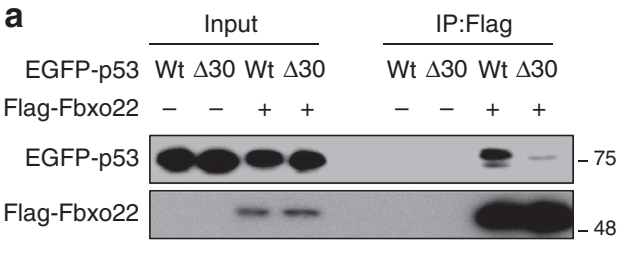

b

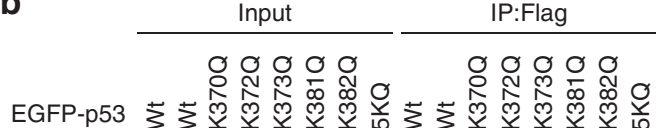

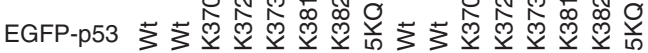

Flag- -+++++++-+++++++

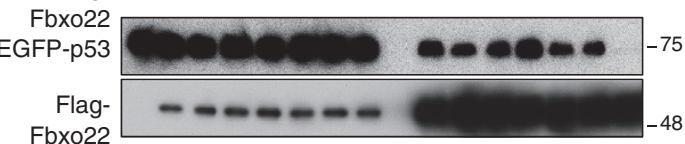

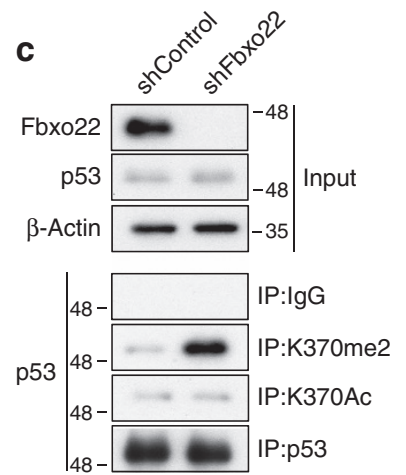

$-75$

Figure 5 | Acetylation of lysines at the CTD of p53 suppresses the interaction with Fbxo22. (a) p53-/- HCT116 cells expressing Dox-inducible Flag-Fbxo22 together with wild-type EGFP-p53 (Wt) or a mutant p53 lacking the C-terminal 30 amino acids ( $\Delta 30$ ) were incubated for $48 \mathrm{~h}$ in the presence or absence of doxycycline $\left(1 \mu \mathrm{g} \mathrm{ml}{ }^{-1}\right)$ and were then treated with MG132 $\left(10 \mu \mathrm{g} \mathrm{ml}^{-1}\right)$ for $2 \mathrm{~h}$. The lysates were immunoprecipitated using anti-Flag M2 affinity gel. The resultant immunoprecipitates and lysates (Input) were subjected immunoblotting using the indicated antibodies. (b) $p 53^{-/-}$HCT116 $^{-1}$ cells expressing Dox-inducible Flag-Fbxo22 together with the wild-type or the indicated mutants of EGFP-p53 were incubated for $48 \mathrm{~h}$ in the presence or absence of doxycycline $\left(1 \mu \mathrm{g} \mathrm{ml}^{-1}\right)$ and were then treated with MG132 $\left(10 \mu \mathrm{g} \mathrm{ml}^{-1}\right)$ for $2 \mathrm{~h}$. The lysates were immunoprecipitated using anti-Flag M2 affinity gel. The resultant immunoprecipitates and lysates (Input) were subjected to immunoblotting using the indicated antibodies. (c) RPE cells expressing the Dox-inducible shControl or shFbxo22 were incubated for $48 \mathrm{~h}$ in the presence of doxycycline $\left(1 \mu \mathrm{g} \mathrm{ml}{ }^{-1}\right)$ and were then treated with MG132 $\left(10 \mu \mathrm{g} \mathrm{ml}^{-1}\right)$ for $2 \mathrm{~h}$. The lysates were immunoprecipitated using a control lgG, methyl (K370me2)- or acetyl (K370Ac)-specific anti-p53, or anti-p53 antibodies. The resultant immunoprecipitates and lysates (Input) were subjected to immunoblotting using the indicated antibodies.

purpose, we induced senescence by specific activation of p53 at G2 phase because this protocol does not cause DNA damage and activate DNA-damage responses, which allowed us to analyse the senescence process more specifically. When Fbxo22 was depleted according to the experimental outline (Fig. 7a), p53 and p21 were readily detectable after removal of Nutlin $3 a$, whereas they were 

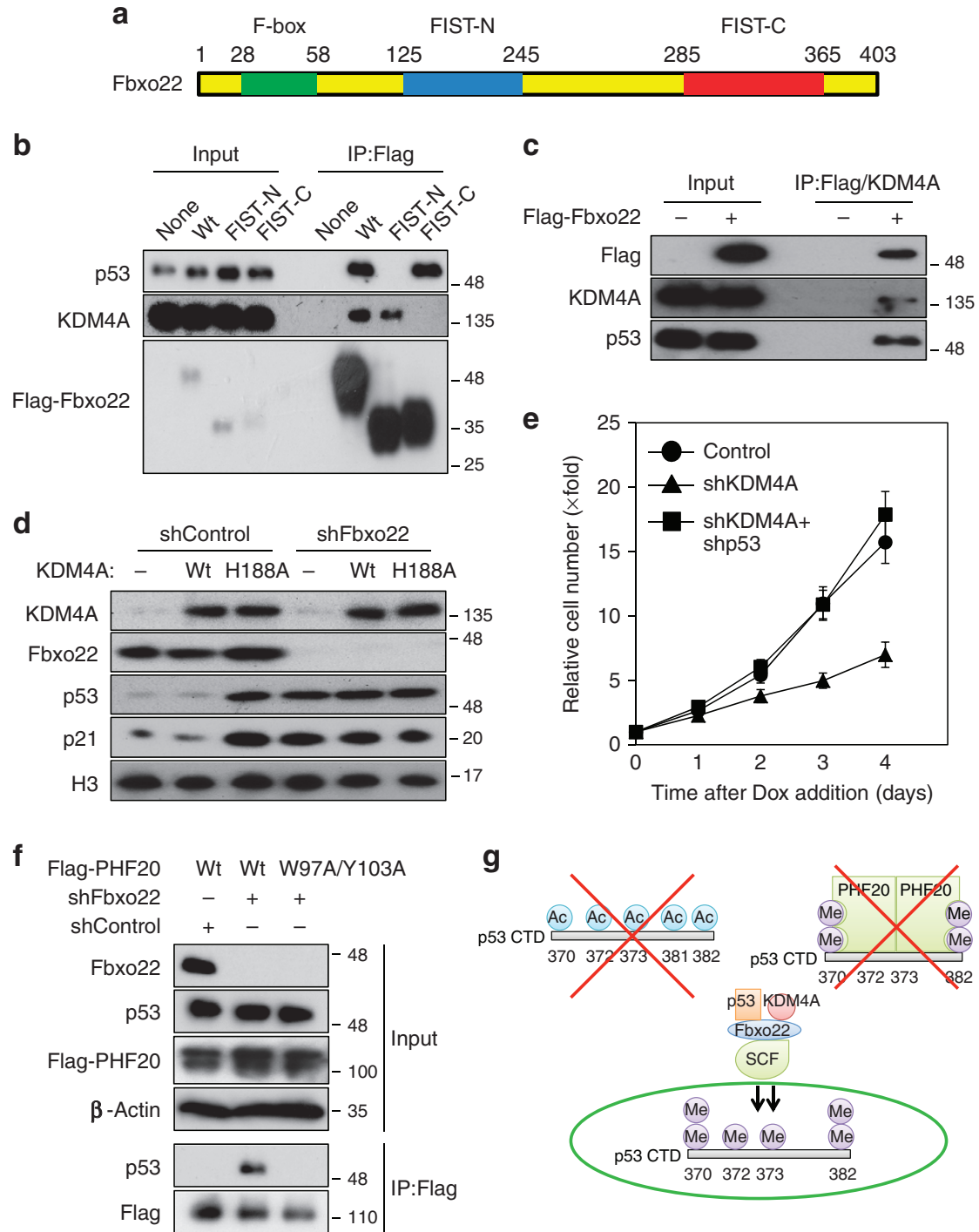

Figure 6 | SCF ${ }^{\mathrm{Fbxo22}}$ forms a ternary complex with p53 and KDM4A that targets methylated p53 for degradation. (a) Schematic representation of the domain structure of Fbxo22. (b) RPE cells expressing Dox-inducible wild-type Flag-Fbxo22 (Wt), and its mutants lacking FIST-N ( $\Delta$ FIST-N) or FIST-C $\left(\Delta\right.$ FIST-C) were incubated for $48 \mathrm{~h}$ in the presence or absence of doxycycline $\left(1 \mu \mathrm{g} \mathrm{ml}^{-1}\right)$ and were then treated with $\mathrm{MG} 132\left(10 \mu \mathrm{g} \mathrm{ml} \mathrm{l}^{-1}\right)$ for $2 \mathrm{~h}$. The lysates were immunoprecipitated using anti-Flag M2 affinity gel. The resultant immunoprecipitates and lysates (Input) were subjected to immunoblotting using the indicated antibodies. (c) RPE cells expressing Dox-inducible Flag-Fbxo22 were incubated for $48 \mathrm{~h}$ in the presence or absence of doxycycline $\left(1 \mu \mathrm{gl}^{-1}\right)$ and were then treated with MG132 $\left(10 \mu \mathrm{g} \mathrm{ml}{ }^{-1}\right)$ for $2 \mathrm{~h}$. The lysates were subjected to sequential immunoprecipitation using anti-Flag $\mathrm{M} 2$ affinity gel and anti-KDM4A antibodies. The resultant immnoprecipitates and lysates were subjected to immunoblotting using the indicated antibodies. (d) RPE cells expressing the Dox-inducible shControl or shFbxo22 together with wild-type KDM4A (Wt) or its catalytically inactive mutant (H188A) were incubated for $48 \mathrm{~h}$ in the presence of doxycycline $\left(1 \mu \mathrm{g} \mathrm{ml}^{-1}\right)$ and were then treated with MG132 $\left(10 \mu \mathrm{g} \mathrm{ml}^{-1}\right)$ for $2 \mathrm{~h}$. The lysates were subjected to immunoblotting using the indicated antibodies. (e) RPE cells expressing the Dox-inducible shControl, shKDM4A or shKDM4A + shp53 were treated with doxycycline $\left(1 \mu \mathrm{g} \mathrm{ml}^{-1}\right)$ and their relative numbers were determined at the indicated times. Data are presented as means $\pm \mathrm{s}$.d. of at least three independent experiments. (f) RPE cells expressing the Dox-inducible shControl or shFbxo22, together with wild-type Flag-PHF20 (Wt) or its WD domain mutant (W97A/Y103A) were incubated for $48 \mathrm{~h}$ in the presence of doxycycline $\left(1 \mu \mathrm{g} \mathrm{ml}^{-1}\right)$ and were then treated with $\mathrm{MG}^{-132}(10 \mu \mathrm{g} \mathrm{ml}-1)$ for $2 \mathrm{~h}$. The cell lysates were immunoprecipitated using anti-Flag M2 affinity gel. The resultant immunoprecipitates and lysates (Input) were subjected to immunoblotting using the indicated antibodies. (g) Schematic representation of CTD modifications that protect p53 from SCF Fbxo22 -targeted degradation.

hardly detectable in control cells (Fig. 7b). Treatment with Nutlin $3 \mathrm{a}$ at G2 phase according to this experimental outline effectively induced senescence phenotypes such as an increased $4 \mathrm{~N}$ population, an increased SA- $\beta$-gal-positive cells, an increased ROS level and cessation of cell proliferation without inducing apoptosis and DNA damage (Supplementary Fig. 8a-f). Induction of p16 and activation of p38MAPK, as evaluated by HSP27 phosphorylation, were greatly suppressed in Fbxo22-depleted Nutlin 3a-induced senescent cells although Fbxo22 depletion did not affect most of the senescent phenotypes such as an increased $4 \mathrm{~N}$ population, an increased in SA- $\beta$-gal-positive cells, a rise in the ROS level, and cessation of cell proliferation induced by this experimental outline (Supplementary Fig. 9a-d). Accordingly, induction of IL-6 and IL-8 was strongly compromised in Fbxo22depleted cells, but not in control cells (Fig. 7c). A requirement for Fbxo22 in the induction of p16 and SASP was also observed in replicative senescent cells (Supplementary Fig. 10a-c) as well as in oncogene-induced senescent cells (Supplementary Fig. 11a-c). In addition, similar results were also observed in KDM4A-depleted Nutlin 3a-induced senescent cells (Fig. 7d,e). Again, KDM4A 


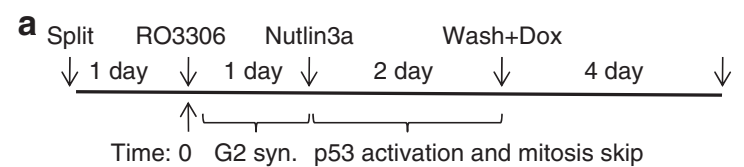

b

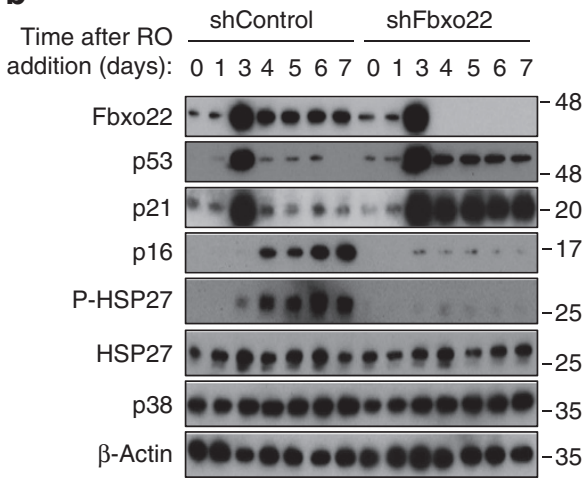

d

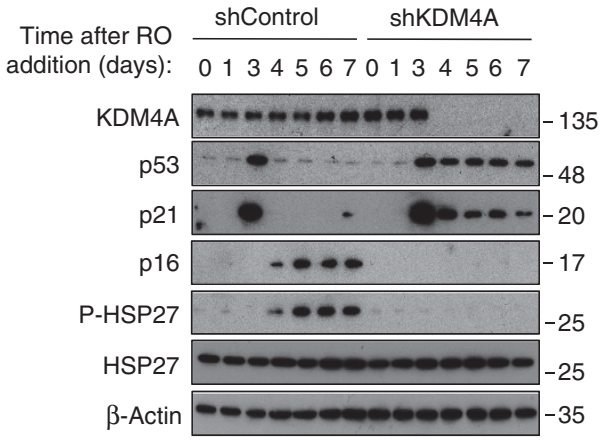

C

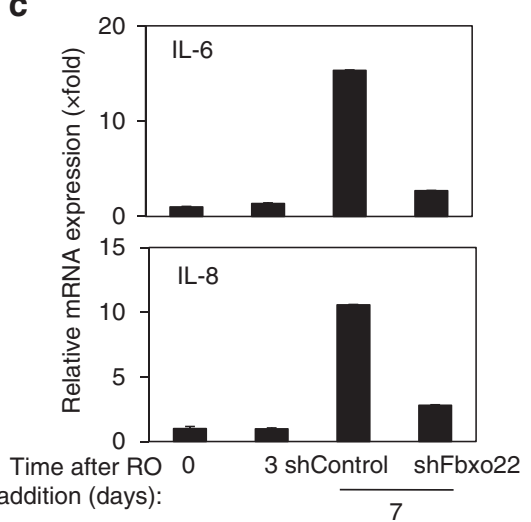

e

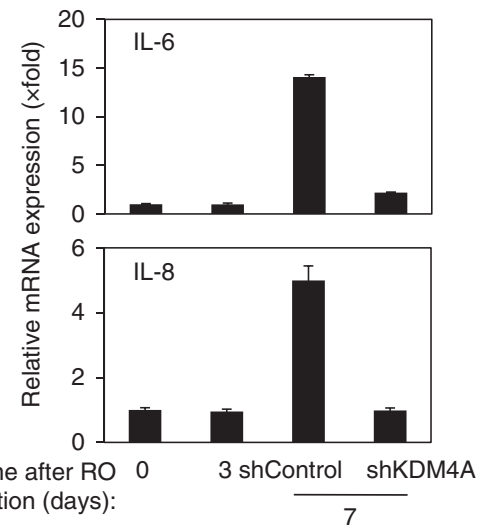

Figure 7 | SCF Fbxo22-KDM4A-dependent degradation of p53 is essential for induction of p16 and SASP in senescent cells. (a) Experimental outline of Fbxo22 or KDM4A depletion in senescent cells. (b) HCA2 cells expressing the Dox-inducible shControl or shFbxo22 treated as in a were subjected to immunoblotting using the indicated antibodies. (c) Total RNAs from HCA2 cells as in $\mathbf{b}$ were subjected to 7 days of qPCR analysis using the indicated primers. (d) HCA2 cells expressing the Dox-inducible shControl or shKDM4A treated as in a were subjected to immunoblotting using the indicated antibodies. (e) Total RNAs from HCA2 cells as in $\mathbf{d}$ were subjected to 7 days of qPCR analysis using the indicated primers. Data are presented as means \pm s.d. of at least three independent experiments.

depletion did not affect most of the senescent phenotypes (Supplementary Fig. 12a-d). The suppression of p16 and SASP induction in senescent cells by continuous activation of $\mathrm{p} 53$ was confirmed by continuous treatment with Nutlin 3a (Supplementary Fig. 13a,b) or Hdm2 depletion (Supplementary Fig. 14a-c). Once again, continuous activation of p53 did not affect most of the senescent phenotypes, including cessation of cell proliferation, an increased ROS level, and an increased in SA- $\beta$-gal-positive cells, whereas it strongly suppressed NF- $\kappa B$ activity which is reported to be essential for SASP $^{9,29}$ (Supplementary Fig. 8a-g). As expected, expression of wildtype, but not W97A/Y103A mutant, PHF20 in Nutlin 3a-induced senescent cells compromised p53 reduction and suppressed p16 and SASP induction, although their expression did not affect most of the senescent phenotypes (Supplementary Fig. 15a-e). Thus, a negative feedback loop between p53 and Fbxo22 plays an important role in induction of p16 and SASP in senescent cells. The $\mathrm{SCF}^{\mathrm{Fbxo22}}$-KDM4A complex appears to be a central player in the induction of senescence phenotypes.

Fbxo22 regulates the abundance of $\mathrm{p} 53$ in vivo. In order to clarify the physiological function of Fbxo22 in p53 regulation, we generated Fbxo22 mutant mice using the CRISPR-Cas9 system ${ }^{30}$. Pronuclear injection of circular plasmids expressing Cas9 and single-guide RNA that targets exon 1 of the Fbxo22 gene resulted in generation of 3 nullizygous $\left(\mathrm{FbxO}^{-1} 2^{-}\right)$and one heterozygous mouse $\left(\mathrm{Fbxo22} 2^{+/-}\right)$, all of which possessed distinct deletions or mutations in exon 1 of the Fbxo22 gene (Supplementary Fig. 16a). Although one Fbxo22 ${ }^{+/-}$mouse appeared normal, two $F b \times 022^{-1-}$ mice were viable but were of smaller size with almost half the body weight at 6 months of age as compared with Fbxo22 $2^{+/-}$or Fbxo22 $2^{+/+}$mice (Fig. 8a). Relative organ weights of these Fbxo22 ${ }^{-/-}$mice were comparable with those of $\mathrm{Fbxo22} 2^{+/-}$or $\mathrm{Fbxo22}^{+/+}$mice (Supplementary Fig. 16b). The other Fbxo22 ${ }^{-1-}$ mouse died within 2 days of birth. In order to eliminate the possibility that the phenotypes observed in Fbxo22 $2^{-/-}$mice were owing to offtarget effects in vivo, we amplified DNA fragments by PCR from all 9 potential off-target loci (Supplementary Fig. 17) predicted by a previously determined recognition rule ${ }^{30}$, and found no mutations, deletions or insertions. Taken together with the fact that all mutant mice possessed distinct mutations or deletions, phenotypes in Fbxo22 $2^{-1-}$ mice were unlikely to have been because of the off-target effects. We intercrossed $\mathrm{Fbxo22}^{+/-}$ 
a

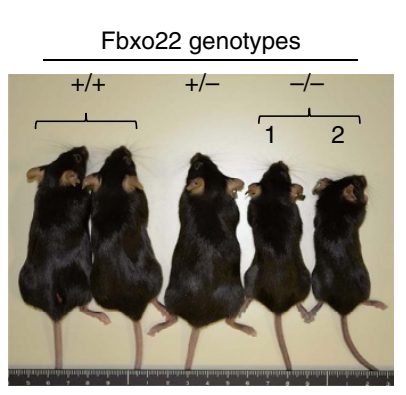

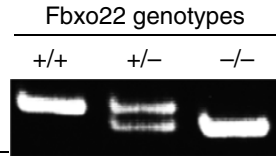

d

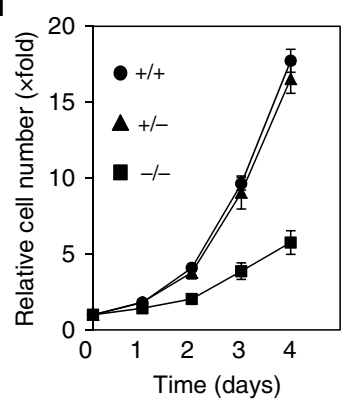

e

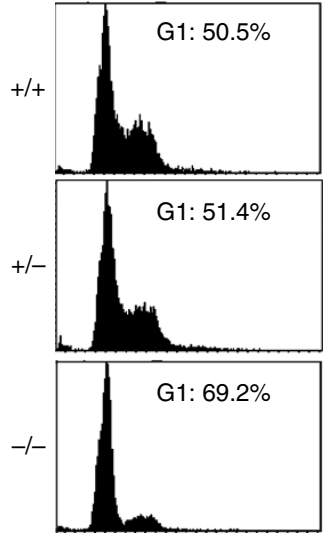

b

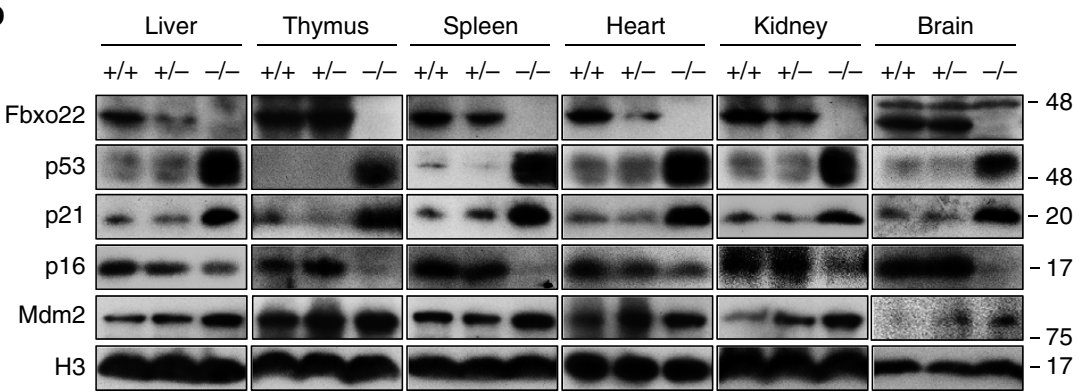

f

g
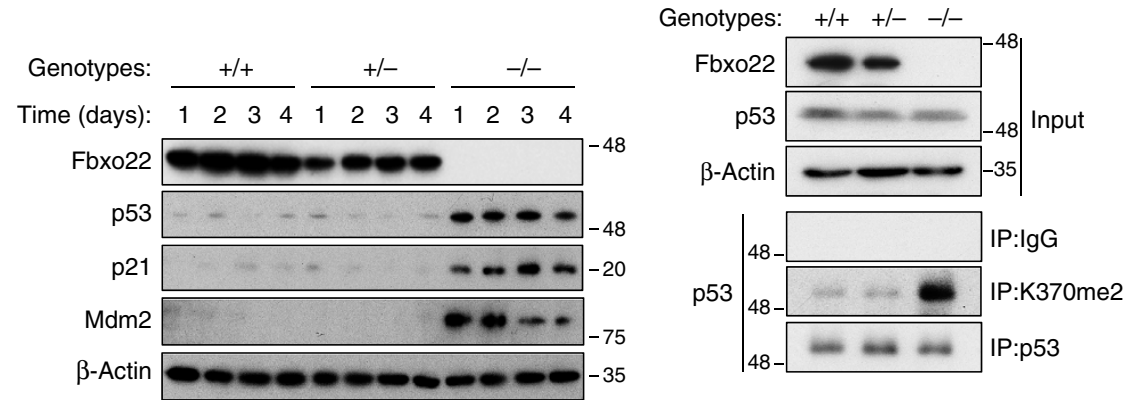

Figure 8 | CRISPR/CAS9-mediated disruption of mouse Fbxo22. (a) Representative Fbxo22 wild-type $(+/+)$, heterozygous $(+/-)$ or nullizygous $(-/-)$ mice at 24 weeks of age. (b) Lysates of tissues from the spleen, thymus, kidney, liver, lung and brain of 24-week-old Fbxo22 wild-type ( $+/+)$, heterozygous $(+/-)$ or nullizygous $(-/-)$ mice were analysed by immunoblotting using the indicated antibodies. (c) Genotype analysis of Fbxo22 wild-type $(+/+)$, heterozygous $(+/-)$ and nullizygous $(-/-)$ mice by PCR. (d) Primary MEFs from Fbxo22 $-/-$ mice showed severe growth retardation. Relative numbers of primary MEFs from Fbxo22 $+/+, F b x 022^{+/-}$and Fbxo22 $-/-$mice were determined at the indicated times. Data are presented as means \pm s.d. of at least three independent experiments. (e) Cell cycle distribution of primary MEFs as in $\mathbf{d}$ was determined at 4 days by FACScan. (f) Primary MEFs from Fbxo22-/- mice showed marked accumulations of p53, p21 and Mdm2. Primary MEFs from mice with the indicated genotypes were collected at the indicated times and the lysates were subjected to immunoblotting as shown. (g) Primary MEFs with the indicated genotypes were treated with MG132 $\left(10 \mu \mathrm{g} \mathrm{ml}^{-1}\right)$ for $2 \mathrm{~h}$. The lysates were immunoprecipitated using a control lgG, methyl (K370me2)-specific anti-p53 or anti-p53 antibodies. The resultant immunoprecipitates and lysates (Input) were subjected to immunoblotting using the indicated antibodies.

mice and found that the genotype distribution of the offspring was consistent with the Mendelian-based ratio of 1:2:1 although some portion of the $F b \times 22^{-/-}$mice died within a couple of days, indicating that, unlike $M d m 2, F b x o 22$ is dispensable for early embryonic development.

p53 and p21 as well as Mdm2 markedly accumulated in all tissues tested in Fbxo22 $2^{-/}$mice (Fig. 8b). Interestingly, the level of p16 in these mice was greatly decreased in all tissues tested, indicating that the role of $\mathrm{SCF}^{\mathrm{Fbxo22}}-\mathrm{KDM} 4 \mathrm{~A}$ in the induction of p16 was also conserved in vivo. Histo-pathological analyses of tissues from $F b x o 22^{-/-}$mice revealed that their anatomical structures appeared normal (data not shown). Mouse embryonic fibroblast (MEFs) from $\mathrm{Fbxo22} 2^{-/-}$mice showed a severe growth defect when compared with those from $\mathrm{Fbxo22}+/+$ or Fbxo22 $2^{+/-}$mice (Fig. 8c,d), owing to the increase in the G1 population (Fig. 8e). As in mice tissues, p53, p21 and Mdm2 markedly accumulated in MEFs from Fbxo22-/-, but not Fbxo $22^{+1+}$ and Fbxo22 $2^{+/-}$mice (Fig. 8f). In addition, methylated p53 was significantly increased in MEFs from Fbxo22 $-1-$ mice, but not Fbxo22 $+1+$ or Fbxo22 $2^{+/-}$mice in the presence of MG132 (Fig. 8g). Ectopically expressed wild-type PHF20, but not its W97A/Y103A mutant, formed a complex with p53 in cells from Fbxo22 $-/-$ mice, but not Fbxo22 $2^{+/+}$and Fbxo22 $+1-$ mice (Supplementary Fig. 18), reflecting the increase 
in the amount of methylated p53 in cells from $\mathrm{Fbxo22}-1-$ mice. These results clearly show that the phenotypes observed in Fbxo22 $2^{-/}$mice were consistent with those in their MEFs.

\section{Discussion}

The functioning of p53 in senescence processes is biphasic; it is essential in the early phase of senescence in mitotic skipping by its phase-specific activation at G2 phase, whereas it must be removed at the late phase for the induction of p16 and SASP to occur. This suggests that the pathways regulating the level and timing of p53 activation are critical for overall senescence regulation. In this study, we showed that the $\mathrm{SCF}^{\mathrm{Fbxo} 22}-\mathrm{KDM} 4 \mathrm{~A}$ complex is an indispensable element through its function as a novel E3 ubiquitin ligase complex targeting methylated p53 for degradation at the late senescent stage.

Over a dozen E3 ubiquitin ligases and modulators have been reported to regulate p53 stability under certain circumstances. However, there is overwhelming molecular and genetic evidence supporting a major role for $\mathrm{Mdm} 2$ in targeting p53 for degradation under both stress and non-stress conditions ${ }^{10,11,14}$ but the in vivo relevance of other E3 ligases in p53 regulation is far less clear. In this respect, we successfully identified the $\mathrm{SCF}^{\mathrm{Fbxo22}}$-KDM4A complex as a bona fide $\mathrm{E} 3$ ligase for $\mathrm{p} 53$. The strongest evidence for this is that target disruption of Fbxo22 in mice and their MEFs resulted in a marked increase in amounts of p53, p21 and Mdm2, but a decrease in p16 (Fig. 8a,b), which resulted in the small body size of these mice and the severe growth defects affecting their MEFs (Fig. 8c-f). Similar phenotypes were observed in $S k p 2^{-1-}$ mice and MEFs in which another Cdk inhibitor, p27, had accumulated ${ }^{31}$. Similar to $\mathrm{Mdm} 2$, the Fbxo22 gene is a direct transcriptional target of p53, suggesting a plausible mechanism by which p53 activity could be reset to basal levels after shutdown of p53-inducible stimuli, although its p53-dependent induction was relatively slower than that of Mdm2 and p21 (Fig. 1d,e and Supplementary Fig. 2). Taken together, the normal p53 degradation profile of mice with a disrupted $\mathrm{p} 53-\mathrm{mdm} 2$ negative feedback loop may be explained in terms of $\mathrm{SCF}^{\mathrm{Fbxo} 22}$-p53 circuitry.

SCF ${ }^{\text {Fbxo22 }}$ bound to the CTD of p53 (Fig. 5a), and unlike $\mathrm{Mdm} 2$, this binding was not affected by $\mathrm{p} 53$ phosphorylation induced by DNA damage (Fig. 4c). In contrast, $\mathrm{SCF}^{\mathrm{Fbxo} 22}$ binding to p53 was affected by acetylation and/or methylation of the lysine cluster at K370-K386 on p53 CTD ${ }^{19}$. Given that acetylated p53 was not a degradation target of $\mathrm{SCF}^{\mathrm{Fbxo22}}-\mathrm{KDM} 4 \mathrm{~A}$ (Fig. 5b), this could effectively explain why acetylation on the p53 CTD by $\mathrm{CBP} / \mathrm{p} 300$ greatly increased p53 stabilization ${ }^{32}$. Although this increased stability might also be reflected by competitive modifications between $\mathrm{CBP} / \mathrm{p} 300$-mediated acetylation and Mdm2-mediated ubiquitylation at the $\mathrm{CTD}^{33}$, this scenario is unlikely because methylation at K372 or K370 by KMT5 or KMT3C did not increase p53 stabilization ${ }^{34}$. In this respect, depletion of Fbxo22 in cells resulted in a specific increase in the abundance of methylated p53, but not acetylated p53 (Fig. 5c) and overexpression of a catalytic mutant, KDM4A, resulted in p53 stabilization (Fig. 6d). Thus, the $\mathrm{SCF}^{\mathrm{Fbxo} 22}-\mathrm{KDM} 4 \mathrm{~A}$ complex likely targets methylated p53 for ubiquitin-dependent degradation, consistent with previous reports showing that most p53 methylation acted as a mark of repression of p53-dependent transcriptional activity ${ }^{19}$. In addition, it should be noted that KDM4A was reported to suppress senescence by negatively regulating the p53 pathway via transcriptional suppression of CHD5 (ref. 35). Our results are also consistent with a recent report that PHF20 leads to p53 stabilization and activation 28 because PHF20 binding to methylated p53 protected it from $\mathrm{SCF}^{\mathrm{Fbxo22}}$-KDM4A-mediated degradation (Fig. 6f). Taken together, the interplay between acetylation and methylation at the p53 CTD directly regulate p53 stability by controlling the access of Fbxo22 to the p53 CTD (Fig. 6g).

Although p53 is essential for triggering senescence induction, subsequent induction of p16 appears critical for maintaining irreversible growth arrest ${ }^{5,36}$. p53 was reported to inhibit p38MAPK, leading to the suppression of SASP phenotypes? Thus, p53 must be downregulated at the late stage of senescence, demonstrating the biphasic functioning of p53. Fine-tuning of this biphasic function is likely achieved by temporal orchestration of p53 ubiquitylation by $\mathrm{Mdm} 2$ and $\mathrm{SCF}^{\mathrm{Fbxo22}}-\mathrm{KDM} 4 \mathrm{~A}$ which regulate early and late events, respectively. Most notably, SCF ${ }^{\mathrm{Fbx} 22}$-KDM4A is essential for SASP induction and could therefore be a promising candidate for the development of drugs targeting cancer as well as geriatric diseases, since cytokines and growth factors secreted from senescent cells have recently been implicated in chronic inflammation and various age-related changes, including carcinogenesis in vivo ${ }^{7,37,38}$.

\section{Methods}

Cell culture. Early passage HCA2 (ref. 39), HeLa (ATCC), U2OS (ATCC), MCF7 (RIKEN Cell Bank) cells, MEFs and HEK-293T (RIKEN Cell Bank) cells were cultured in DMEM (Invitrogen) supplemented with $10 \%$ fetal bovine serum (FBS). RPE-1 (ATCC) were cultured in DMEM/F-12 (Invitrogen) supplemented with non-heat-inactivated 10\% FBS. HCT116 cells were grown in McCoy's 5A (Gibco) supplemented with FBS. Cells were synchronized at G2 phase by treatment with $9 \mu \mathrm{M}$ of RO3306 (Roche) for $24 \mathrm{~h}$. Nutlin 3a (Sigma-Aldrich), cycloheximide (Sigma-Aldrich), trichostatin A or MG132 (Sigma-Aldrich) was used at a concentration of $5 \mu \mathrm{M}, 50 \mu \mathrm{g} \mathrm{ml}^{-1}, 1 \mathrm{mM}$ or $10 \mu \mathrm{g} \mathrm{ml}^{-1}$, respectively, for the specified intervals.

Plasmid construction. To generate lentivirus-based shRNA constructs, a 19-21 bp shRNA-coding fragment with a 5'-ACGTGTGCTGTCCGT-3' loop was introduced into pENTR4-H1 digested with AgeI/EcoRI. To insert the H1tetOx1-shRNA into lentivirus vector, we mixed the resulting pENTR4-H1shRNA vector and CS-RfA-ETBsd vector with Gateway LR clonase (Invitrogen). All the target sequences for lentivirus-based sh-RNAs are summarized in Supplementary Table 1 . To construct Tet-on-inducible lentivirus constructs, the PCR-generated BamHI/Not I fragments containing cDNA for human/mouse Fbxo22 wild type or the respective mutants, human KDM4A wild type or $\mathrm{H} 188 \mathrm{~A}$ and human p53 wild type or the respective mutants, were inserted into a pENTR-1A vector (Invitrogen) containing the Flag epitope or EGFP digested with BamHI/NotI. The resultant plasmid was mixed with CS-IV-TRE-RfA-UbC-Puro or CS-IV-TRE-RfA-UbC-Hygro vector, and reacted with Gateway LR clonase to generate the lentivirus plasmid. Plasmids expressing $h$ Cas 9 and sgRNA were prepared by ligating oligos for the targeting site (mouse Fbxo22 exon1-163-185) into the BbsI site of pX330. The pcDNA3-HA-KDM4A, pcDNA-3-FLAG-PHF20 and pX330 plasmids were kindly provided by Dr Kristian Helin, Dr Mark Bedford and Feng Zhang, respectively.

FACS analysis and FACS sorting. For FACS sorting, HCA2 cells treated with IR (10 Gy) were sorted according to size and structure (FSC and SSC) using a BD FACSAria-2 cell sorter (BD Biosciences). For cell cycle profiles, cells were collected and fixed with $70 \%$ ethanol. Cells were then washed once with phosphate-buffered saline (PBS), treated with RNase and stained with propidium iodide. Flow cytometry was performed using a FACS CANTO2 flow cytometer (BD Biosciences).

Microarray analysis. Total RNA was prepared using ISOGEN II (Nippongene). RNA hybridization, washing and analysis were performed using SurePrint G3 Human GE $8 \times 60 \mathrm{k}$ version 2.0 (Agilent Technologies) and GeneSpring GX version 12.6.0 (Agilent Technologies).

Virus generation and infection. Lentiviruses expressing the respective shRNAs or genes were generated by co-transfection of $293 \mathrm{~T}$ cells with pCMV-VSV-G-RSVRevB, pCAG-HIVgp and the respective CS-RfA-ETBsd, CS-RfA-ETHygro, CS-RfA-ETPuro, CS-IV-TRE-RfA-UbC-Puro or CS-IV-TRE-RfA-UbC-Hygro using the calcium phosphate co-precipitation method. Cells infected with the indicated viruses were treated with $10 \mu \mathrm{g} \mathrm{ml}^{-1}$ of blasticidin (Invitrogen), and/or $2 \mu \mathrm{g} \mathrm{ml}^{-1}$ of puromycin (Sigma-Aldrich) for 2-3 days. Doxycycline (Sigma-Aldrich) was added to the medium at a concentration of $1 \mu \mathrm{g} \mathrm{ml}^{-1}$ for inducible expression of the respective shRNAs or genes. 
Immunoprecipitation and immunoblotting analyses. Cells were lysed in TBSN buffer (20 mM Tris-Cl (pH 8.0), $150 \mathrm{mM} \mathrm{NaCl}, 0.5 \%$ NP-40, 5 mM EGTA, $1.5 \mathrm{mM}$ EDTA, $0.5 \mathrm{mM} \mathrm{Na}_{3} \mathrm{VO}_{4}$ and $20 \mathrm{mM} p$-nitrophenylphosphate). The resulting lysates were clarified by centrifugation at $15,000 \mathrm{~g}$ for $20 \mathrm{~min}$ at $4{ }^{\circ} \mathrm{C}$ before immunoprecipitation with the specified antibody. For whole lysates, cells or tissues were directly lysed with Laemmli-buffer (2\% SDS, $10 \%$ glycerol, $5 \%$ 2 -mercaptoethanol, $0.002 \%$ bromophenol blue and $62.5 \mathrm{mM}$ Tris- $\mathrm{HCl}$ at $\mathrm{pH} 6.8$ ). The whole lysates $(20-50 \mu \mathrm{g})$ were separated by SDS-polyacrylamide gel electrophoresis (SDS-PAGE), transferred to a polyvinylidene difluoride(Immobilon-P; Millipore) membrane, and then subjected to immunoblotting with the indicated antibodies using the enhanced chemiluminescence-detection system. All antibodies used in this study are listed in Supplementary Table 2. Full scans of the most important blots are provided in the Supplementary Fig. 19.

\section{Quantitative RT-PCR. Total RNA was extracted using ISOGEN II (Wako)} according to the manufacturer's instructions. For qRT-PCR analysis, cDNAs were synthesized using a SuperScript II cDNA synthesis kit (Invitrogen). Real-time PCR amplifications were performed in 96-well optical reaction plates with Power SYBR Green PCR Master Mix (Applied Biosystems). The relative expression values of each gene were determined by normalization to GAPDH expression for each sample. Primer sequences: p53-Fw ( $5^{\prime}$-TCAACAAGATGTTTTGCCAACTG- $\left.3^{\prime}\right)$, p53-Rv (5'-ATGTGCTGTGACTGCTTGTAGATG-3'), p21-Fw (5'-TCAGGGTCG AAAACGGCG-3'), p21-Rv (5'-AAGATCAGCCGGCGTTTGGA-3'), Fbxo22-Fw (5'-CTCACTGAAGTAGGTCTTTTAG-3'), Fbxo22-Rv (5'-CCAGCCAAGATGA TATTCATATC- $\left.3^{\prime}\right)$, Hdm2-Fw (5'-ACCTCACAGATTCCAGCTTCG- $\left.3^{\prime}\right)$, Hdm2-Rv (5'-TTTCATAGTATAAGTGTCTTTTT-3'), GAPDH-Fw (5'-GAGTCA ACGGATTTGGTC GT- $3^{\prime}$ ), GAPDH-Rv (5'-TTGATTTTGGAGGGATCTCG-3'), IL-6-Fw (5'-CCAGGAGCCCAGCTATGAAC-3'), IL-6-Rv (5'-CCCAGGGAGAA GGCAACTG-3'), IL-8-Fw (5'-AAGGAAAACTGGGTGCAGAG-3'), IL-8-Rv (5'-ATTGCATCTGGCAACCCTAC-3')

SA- $\beta$-gal staining. SA- $\beta$-gal staining was performed as previously described ${ }^{40}$. Cells were washed in PBS, fixed for $5 \mathrm{~min}$ in $2 \%$ formaldehyde $/ 0.2 \%$ glutaraldehyde, washed and incubated at $37^{\circ} \mathrm{C}$ with fresh SA- $\beta$-gal stain solution ( $1 \mathrm{mg}$ of X-gal per $\mathrm{ml} / 40 \mathrm{mM}$ citric acid/sodium phosphate, $\mathrm{pH} 6.0 / 5 \mathrm{mM}$ potassium ferrocyanide $/ 5 \mathrm{mM}$ potassium ferricyanide $/ 150 \mathrm{mM} \mathrm{NaCl} / 2 \mathrm{mM} \mathrm{MgCl}$ ) for $24 \mathrm{~h}$. After staining, cells were washed in PBS, fixed in methanol for $3 \mathrm{~min}$ and then washed in PBS.

ChIP-sequence analysis of the Fbxo22 promoter. Cells were fixed in $1 \%$ formaldehyde for $10 \mathrm{~min}$ at room temperature, followed by 5 min quenching with $125 \mathrm{mM}$ glycine. Cells were washed twice with PBS and cell pellets were flash frozen in liquid nitrogen. The frozen crosslinked pellets were stored at $-80^{\circ} \mathrm{C}$. Cell pellets were resuspended in sonication buffer $(10 \mathrm{mM}$ Tris- $\mathrm{HCl}, \mathrm{pH} 7.5$, $150 \mathrm{mM} \mathrm{NaCl}, 1 \mathrm{mM}$ EDTA, 1\% SDS) and sonicated for 5 cycles of $1 \mathrm{~min}$ each on ice. Sepharose and magnetic beads were coupled to $2 \mu \mathrm{g}$ of the indicated antibodies. Sonicated lysates were incubated overnight at $4{ }^{\circ} \mathrm{C}$ with the antibody-bound beads. Beads were washed once with IP dilution buffer $(20 \mathrm{mM}$ Tris-HCl, $\mathrm{pH} 7.5,150 \mathrm{mM}$ $\mathrm{NaCl}, 1 \mathrm{mM}$ EDTA, $1 \%$ Triton X-100), once with wash buffer 1 (20 mM Tris-HCl, pH 7.5, $500 \mathrm{mM} \mathrm{NaCl}, 2 \mathrm{mM}$ EDTA, $1 \%$ Triton X-100, 0.1\% SDS) and once with wash buffer $2(10 \mathrm{mM}$ Tris- $\mathrm{HCl}, \mathrm{pH} 7.5,0.25 \mathrm{M} \mathrm{LiCl}, 1 \mathrm{mM}$ EDTA, $0.5 \%$ $\mathrm{Na}$-Deoxycholate, $0.5 \% \mathrm{NP}-40)$. The reversal of crosslinkage $\left(65^{\circ} \mathrm{C}\right.$ overnight in the presence of Proteinase K), was followed by RNase A treatment and DNA was purified by phenol-chloroform extraction and ethanol precipitation. ChIP-seq libraries for Illumina sequencing were prepared following the Illumina Truseq ChIP sample Prep Kit protocol. ChIP libraries were sequenced using an Illumina GAII sequencer for 36 bases in single-read mode.

ChIP-seq data processing. High-throughput sequencing of the ChIP fragments was performed using an Illumina Genome Analyzer (Illumina) following the manufacturer's protocol. Sequences were mapped to the human reference genome (NCBI Build 36, hg18) using ELAND software (Illumina). As a result, 26,730,379, $17,020,836,22,559,531,8,296,440,9,319,944,29,174,994,27,258,981,15,964,489$, $15,142,757,27,522,467,24,557,266,9,486,765$ and $11,280,300$ reads were uniquely mapped for p53, K4me3 (5FU 0, 9 h), K4mel (5FU 0, 9 h), K27ac (5FU 0, 9 h), Pol2 (5FU 0, $9 \mathrm{~h}$ ), Pol2 (5FU 0, $9 \mathrm{~h}$ ), $p 53^{-/-} \mathrm{K} 27 \mathrm{ac}(5 \mathrm{FU} 0,9 \mathrm{~h})$ and $p 53^{-/-}$Pol2 (5FU 0,9h), respectively. The ChIP signal values (ChIP counts/expected) were generated as described ${ }^{41}$.

Ubiquitination assay. Using previously described methods ${ }^{42}$, with some modifications, plasmids were transiently transfected into U2OS cells using $\mathrm{X}$-tremeGENE 9. After $43 \mathrm{~h}$, cells were treated with $20 \mu \mathrm{M} \mathrm{MG132}$ for $5 \mathrm{~h}$, and subsequently lysed in lysis buffer $(50 \mathrm{mM}$ Tris- $\mathrm{HCl}, \mathrm{pH} 7.5,300 \mathrm{mM} \mathrm{NaCl}$ and $0.5 \%$ Triton X-100) containing protease inhibitors and deubiquitinase inhibitor. Total cell lysates were immunoprecipitated with antibodies against anti-Myc followed by immunoblotting with HRP-conjugated antibodies against anti-Myc or anti-Ubiquitin. Moreover, to prevent the detection of ubiquitylation of both E3 ligase itself and p53-associated proteins, a ubiquitylation assay was also performed under denaturing conditions. According to the previous methods, cell lysates from transfected cells were lysed with lysis buffer and added to an equal volume of $2 \times$ SDS-PAGE sample buffer and incubated at $100^{\circ} \mathrm{C}$ for $8 \mathrm{~min}$ before being incubated with an anti-p53 antibody and protein G-Sepharose $4 \mathrm{FF}$ at $4{ }^{\circ} \mathrm{C}$. Immunoprecipiated samples were analysed by immunoblotting with HRPconjugated anti-Myc antibody to detect ubiquitylated p53.

Generation of Fbxo22 knock-out mice. Four-week-old C57BL/6N female mice were superovulated and mated with 7 -week-old C57BL/6N males, and the fertilized eggs were collected from the oviducts. The pronuclear stage eggs were injected with pX330-mFbxo22-4 plasmid at $5 \mathrm{ng}^{-1}$. The eggs were cultivated in KSOM overnight, then transferred into the oviducts of 5-week-old pseudopregnant ICR females. Potential off-target sites were found using Optimized CRISPR design software (http://crispr.mit.edu/). The $\sim 500 \mathrm{bp}$ genomic fragments containing the target or off-target in the center were PCR-amplified and sequenced. All experimental procedures conformed to the Regulations for Animal Experimentation at Nagoya City University, and were reviewed by the Institutional Laboratory Animal Care and Use Committee of Nagoya City University and finally approved by the provost. Primary MEFs were derived from E14.5 embryos of 12-week-old double-heterozygote breeders.

\section{References}

1. Smith, J. R. \& Pereira-Smith, O. M. Replicative senescence: implications for in vivo aging and tumor suppression. Science 273, 63-67 (1996).

2. Blackburn, E. H. Switching and signaling at the telomere. Cell 106, 661-673 (2001).

3. Shay, J. W. \& Wright, W. E. Aging. When do telomeres matter? Science 291, 839-840 (2001).

4. Kuilman, T., Michaloglou, C., Mooi, W. J. \& Peeper, D. S. The essence of senescence. Genes Dev. 24, 2463-2479 (2010).

5. Johmura, Y. et al. Necessary and sufficient role for a mitosis skip in senescence induction. Mol. Cell 55, 73-84 (2014).

6. Krenning, L., Feringa, F. M., Shaltiel, I. A., van den Berg, J. \& Medema, R. H Transient activation of p53 in G2 phase is sufficient to induce senescence. Mol. Cell 55, 59-72 (2014).

7. Tchkonia, T., Zhu, Y., van Deursen, J., Campisi, J. \& Kirkland, J. L. Cellular senescence and the senescent secretory phenotype: therapeutic opportunities. J. Clin. Invest. 123, 966-972 (2013).

8. Demaria, M. et al. An essential role for senescent cells in optimal wound healing through secretion of PDGF-AA. Dev. Cell 31, 722-733 (2014).

9. Freund, A., Patil, C. K. \& Campisi, J. p38MAPK is a novel DNA damage response-independent regulator of the senescence-associated secretory phenotype. EMBO J. 30, 1536-1548 (2011)

10. Hock, A. K. \& Vousden, K. H. The role of ubiquitin modification in the regulation of p53. Biochim. Biophys. Acta 1843, 137-149 (2014).

11. Pant, V. \& Lozano, G. Limiting the power of p53 through the ubiquitin proteasome pathway. Genes Dev. 28, 1739-1751 (2014).

12. Brooks, C. L. \& Gu, W. The impact of acetylation and deacetylation on the p53 pathway. Protein Cell 2, 456-462 (2011).

13. Brooks, C. L. \& Gu, W. p53 regulation by ubiquitin. FEBS Lett. 585, 2803-2809 (2011).

14. Love, I. M. \& Grossman, S. R. It takes 15 to tango: making sense of the many ubiquitin ligases of p53. Genes Cancer 3, 249-263 (2012).

15. Prives, C. Signaling to p53: breaking the MDM2-p53 circuit. Cell 95, 5-8 (1998)

16. Fahraeus, R. \& Olivares-Illana, V. MDM2's social network. Oncogene 33, 4365-4376 (2014).

17. Hu, W., Feng, Z. \& Levine, A. J. The regulation of multiple p53 stress responses is mediated through MDM2. Genes Cancer 3, 199-208 (2012).

18. Pant, V. et al. The p53-Mdm2 feedback loop protects against DNA damage by inhibiting p53 activity but is dispensable for p53 stability, development, and longevity. Genes Dev. 27, 1857-1867 (2013).

19. Marouco, D., Garabadgiu, A. V., Melino, G. \& Barlev, N. A. Lysine-specific modifications of p53: a matter of life and death? Oncotarget 4, 1556-1571 (2013).

20. Meek, D. W. Mechanisms of switching on p53: a role for covalent modification? Oncogene 18, 7666-7675 (1999).

21. Tang, Y., Zhao, W., Chen, Y., Zhao, Y. \& Gu, W. Acetylation is indispensable for p53 activation. Cell 133, 612-626 (2008).

22. Chuikov, S. et al. Regulation of p53 activity through lysine methylation. Nature 432, 353-360 (2004)

23. Morgunkova, A. \& Barlev, N. A. Lysine methylation goes global. Cell Cycle 5, 1308-1312 (2006).

24. Vrba, L., Junk, D. J., Novak, P. \& Futscher, B. W. p53 induces distinct epigenetic states at its direct target promoters. BMC Genomics 9, 486 (2008).

25. Tan, M. K., Lim, H. J. \& Harper, J. W. SCF(FBXO22) regulates histone H3 lysine 9 and 36 methylation levels by targeting histone demethylase KDM4A for ubiquitin-mediated proteasomal degradation. Mol. Cell Biol. 31, 3687-3699 (2011). 
26. Pickart, C. M. Targeting of substrates to the $26 \mathrm{~S}$ proteasome. FASEB J. 11, 1055-1066 (1997).

27. Nash, P. et al. Multisite phosphorylation of a CDK inhibitor sets a threshold for the onset of DNA replication. Nature 414, 514-521 (2001).

28. Cui, G. et al. PHF20 is an effector protein of p53 double lysine methylation that stabilizes and activates p53. Nat. Struct. Mol. Biol. 19, 916-924 (2012).

29. Chien, Y. et al. Control of the senescence-associated secretory phenotype by NF-kappaB promotes senescence and enhances chemosensitivity. Genes Dev. 25, 2125-2136 (2011)

30. Wang, H. et al. One-step generation of mice carrying mutations in multiple genes by CRISPR/Cas-mediated genome engineering. Cell 153, 910-918 (2013).

31. Nakayama, K. et al. Targeted disruption of Skp2 results in accumulation of cyclin E and p27(Kip1), polyploidy and centrosome overduplication. EMBO J. 19, 2069-2081 (2000).

32. Gu, W. \& Roeder, R. G. Activation of p53 sequence-specific DNA binding by acetylation of the p53 C-terminal domain. Cell 90, 595-606 (1997).

33. Rodriguez, M. S., Desterro, J. M., Lain, S., Lane, D. P. \& Hay, R. T. Multiple $\mathrm{C}$-terminal lysine residues target p53 for ubiquitin-proteasome-mediated degradation. Mol. Cell Biol. 20, 8458-8467 (2000).

34. Ivanov, G. S. et al. Methylation-acetylation interplay activates p53 in response to DNA damage. Mol. Cell Biol. 27, 6756-6769 (2007).

35. Mallette, F. A. \& Richard, S. JMJD2A promotes cellular transformation by blocking cellular senescence through transcriptional repression of the tumor suppressor CHD5. Cell Rep. 2, 1233-1243 (2012).

36. Beausejour, C. M. et al. Reversal of human cellular senescence: roles of the p53 and p16 pathways. EMBO J. 22, 4212-4222 (2003).

37. Rodier, F. \& Campisi, J. Four faces of cellular senescence. J. Cell Biol. 192, 547-556 (2011)

38. Campisi, J. \& d'Adda di Fagagna, F. Cellular senescence: when bad things happen to good cells. Nat. Rev. Mol. Cell Biol. 8, 729-740 (2007).

39. Nakanishi, M. et al. Exit from G0 and entry into the cell cycle of cells expressing p21Sdil antisense RNA. Proc. Natl Acad. Sci. USA 92, 4352-4356 (1995).

40. Dimri, G. P. et al. A biomarker that identifies senescent human cells in culture and in aging skin in vivo. Proc. Natl Acad. Sci. USA 92, 9363-9367 (1995).

41. Wakabayashi, K. et al. The peroxisome proliferator-activated receptor gamma/retinoid $\mathrm{X}$ receptor alpha heterodimer targets the histone modification enzyme PR-Set7/Setd8 gene and regulates adipogenesis through a positive feedback loop. Mol. Cell Biol. 29, 3544-3555 (2009).

42. Nakajima, T. et al. Regulation of GATA-binding protein 2 levels via ubiquitin-dependent degradation by Fbw7: involvement of cyclin B-cyclin-dependent kinase 1-mediated phosphorylation of THR176 in gata-binding protein 2. J. Biol. Chem. 290, 10368-10381 (2015).

\section{Acknowledgements}

We are grateful to Drs Kristian Helin, Feng Zhang, Mark Bedford, Yasumichi Inoue and Masahito Ikawa for reagents. We are also indebted to Drs Chisato Yamada-Namikawa, Midori Shimada, Dr Kazuhiro Murata, Mr Toshinori Misaki, and the other members of Nakanishi's laboratory for technical assistance. M.N. was supported by a Grant-in-Aid for Scientific Research on Innovative Area 'Cell Fate Control', Scientific Research, and Challenging Exploratory Research from MEXT Japan. Y.J. was supported by a grant for Young Scientific Research.

\section{Author contributions}

M.N. conceived the project. Y.J., J.S., K.K., T.K., K.N., Y.S., T.M., A.O., H.A., S.L., I.M. S.T., M.K., and M.N. designed the experiments. Y.J. and J.S. performed the main experiments and analysed the data. K.K. and M.K. performed the ubiquitination assays Y.J., K.N., T.M., and I.M. generated and analysed the protein levels of tissues in the Fbxo22 $2^{-/-}$mice. T.K., A.N.-I., and S.T. analysed the histopathology of Fbxo22-/mice. A.O. and H.A. performed ChIP-seq analysis of the Fbxo22 gene. M.N. wrote the manuscript and directed the study. All authors were involved in discussions of the manuscript.

\section{Additional information}

Accession codes: Microarray expression profiling was performed by Oncomics (Nagoya, Japan), GEO accession: GSE69116.

Supplementary Information accompanies this paper at http://www.nature.com/ naturecommunications

Competing financial interests: The authors declare no competing financial interests.

Reprints and permission information is available online at http://npg.nature.com/ reprintsandpermissions/

How to cite this article: Johmura, Y. et al. $\mathrm{SCF}^{\mathrm{Fbxo22}}$-KDM4A targets methylated p53 for degradation and regulates senescence. Nat. Commun. 7:10574 doi: $10.1038 /$ ncomms10574 (2016)

(c) (i) This work is licensed under a Creative Commons Attribution 4.0 cc) International License. The images or other third party material in this article are included in the article's Creative Commons license, unless indicated otherwise in the credit line; if the material is not included under the Creative Commons license, users will need to obtain permission from the license holder to reproduce the material. To view a copy of this license, visit http://creativecommons.org/licenses/by/4.0/ 